\title{
UCRL-JRNL-204384
}

LAW RENCE LIVERMORE N A TIO NAL LABORATORY

\section{A "First Principles" Potential Energy Surface for Liquid Water from VRT Spectroscopy of Water Clusters}

N. Goldman, C. Leforestier, R. J. Saykally

May 27, 2004

Philosophical Transactions of the Royal Society A 
This document was prepared as an account of work sponsored by an agency of the United States Government. Neither the United States Government nor the University of California nor any of their employees, makes any warranty, express or implied, or assumes any legal liability or responsibility for the accuracy, completeness, or usefulness of any information, apparatus, product, or process disclosed, or represents that its use would not infringe privately owned rights. Reference herein to any specific commercial product, process, or service by trade name, trademark, manufacturer, or otherwise, does not necessarily constitute or imply its endorsement, recommendation, or favoring by the United States Government or the University of California. The views and opinions of authors expressed herein do not necessarily state or reflect those of the United States Government or the University of California, and shall not be used for advertising or product endorsement purposes. 


\title{
A "First Principles" Potential Energy Surface for Liquid Water from VRT Spectroscopy of Water Clusters
}

\author{
Nir Goldman, ${ }^{\dagger}$ Claude Leforestier,${ }^{\S}$ and R. J. Saykally ${ }^{\ddagger * *}$
}

Lawrence Livermore National Laboratory, Chemistry and Materials Science Directorate, L-268, Livermore, California 94551, LSDMS (UMR 5636), CC 014, Université Montpellier II, 34095

Montpellier Cédex, France, Department of Chemistry, University of California, Berkeley, California, 94720-1416.

\begin{abstract}
We present results of gas phase cluster and liquid water simulations from the recently determined VRT(ASP-W)III water dimer potential energy surface. VRT(ASP-W)III is shown to not only be a model of high "spectroscopic" accuracy for the water dimer, but also makes accurate predictions of vibrational ground-state properties for clusters up through the hexamer. Results of ambient liquid water simulations from VRT(ASP-W)III are compared to those from ab initio Molecular Dynamics, other potentials of "spectroscopic" accuracy, and to experiment. The results herein represent the first time that a "spectroscopic" potential surface is able to correctly model condensed phase properties of water.
\end{abstract}

\section{Introduction}

Literally hundreds of effective potential energy surfaces have now been developed for the purpose of computing properties of liquid water and aqueous solutions via classical (Molecular Dynamics or

\footnotetext{
†Lawrence Livermore National Laboratory, Chemistry and Materials Science Directorate, L-268, Livermore, California 94551

$\S$ LSDMS (UMR 5636), CC 014, Université Montpellier II, 34095 Montpellier Cédex, France

$\ddagger$ Department of Chemistry, University of California, Berkeley, California, 94720-1416

*Corresponding author. Email: saykally@uclink4.berkeley.edu.
} 
Monte Carlo) simulations. While the use of these surfaces has engendered much progress in understanding the properties of aqueous systems, there always remains a degree of doubt concerning the validity of simulations results because of the simplistic and empirical nature of these potential surfaces [1]. This is particularly the case when properties are computed outside the range of conditions for which the effective surfaces were parameterized [1].

These empirical potential surfaces are generally formulated by using classical simulations to adjust a simple analytical expression to structural and thermodynamic properties of bulk water (c. f., $[2,3]$ ). Accordingly, quantum effects (tunneling through potential barriers to hydrogen bond rearrangement, zero-point energies) are not properly represented, and the corresponding IPS are artificially "smoothed", relative to the topology of the true quantum surface (i. e with the actual potential barriers and deeper potential wells). Hence, it is not surprising that quantum simulations employing such classical effective potentials yield the conclusion that quantum effects in ambient liquid water are small, equivalent to simply raising the temperature in a classical simulation by $50^{\circ} \mathrm{C}[4,5]$.

The development of $a b$ initio molecular dynamics methods has been a great advance in the study of condensed matter systems, mitigating the above concerns regarding the use of effective potentials. However, these involve the use of fully quantum potentials in classical simulations, and might therefore underestimate those dynamics for which sizable potential barriers exist in the pathway, e.g. hydrogen bond rearrangement in liquid water. Moreover, their dependence on density functional theory methods for computing the electronic energy, and the resulting difficulty in determining nonlocal interactions (dispersion) [6] engenders an element of uncertainty in calculations of systems for which such interactions are known to be important (n. b., dispersion constitutes ca. $20 \%$ of the cohesive energy of water [7]). Finally, concerns have been raised regarding the convergence of $a b$ initio MD methods [8]. These facts make it clear that further study of the water force field is in order. 


\section{Determination of the $\left(\mathrm{H}_{2} \mathrm{O}\right)_{2}$ potential surface}

In order to address these issues, we have taken a bottom up "first principles" approach in which we have created a liquid potential through highly accurate determination of dimer properties. In a recent paper, Hodges et al. calculate the total ab initio interaction energies for the water trimer, tetramer and pentamer and dissect them into their respective N-body components [9]. The results show that the 2 -body forces comprise ca. $75 \%$ of the total energy, the 3 -body terms ca. $20 \%$, and the $4-5$-body terms the remaining $5 \%$. In a more detailed paper, Ojäme and Hermansson performed a similar analysis of many-body forces operative in chains of water molecules up to the heptamer, in ring structures up to the pentamer, and in a tetrahedral pentamer [10]. These calculations, performed at the MP2 level, yield some striking insights. In both the water heptamer chain and the pentamer ring structure, they find that 2-body forces account for over $80 \%$ of the total interaction energy, and that two- and three-body terms together account for over $99 \%$. In the tetrahedral pentamer, which closely resembles the average liquid and normal ice structures, they find that the two-body energy constitutes over $87 \%$ of the total interaction energy, and the two- and three-body terms together comprise ca. 99.6\%. In all cases, the total energies of larger clusters are rapidly converging and are essentially fully converged by accounting for only the two- and three-body terms. Hence, rigorous description of the pairwise interaction appears to be the central issue for constructing a complete molecular (i. e., non-empirical) description of the liquid.

A series of new IPS, formulated by refining ab initio-based analytical surfaces against the extensive VRT spectroscopy data now available for the water dimer, has been reported by two groups. One of the most accurate water dimer potentials obtained to date is the recently determined VRT(ASPW)III water dimer intermolecular potential energy surface (IPS) [11]. This is the third fitting of Millot and Stone's rigid ASP-W ab initio potential [12] to $\left(\mathrm{D}_{2} \mathrm{O}\right)_{2}$ intermolecular vibration-rotation (VRT) tunneling transitions. The dimer tunneling splittings resulting from hydrogen bond rearrangements and the intermolecular vibrational frequencies provide a highly sensitive probe of the complex water intermolecular potential energy surface (IPS) [13], and such measurements have been made extensively by our laboratory [14-16]. The ASP-W potential has 72 parameters, corresponding to electrostatic interactions, two-body exchange-repulsion, two-body dispersion, and many-body induction, but it was found previously that accurate fits to the data could be produced by fitting 
just a few of the 22 exchange-repulsion parameters [17]. Thus, the VRT(ASP-W)III potential was generated by fitting 6 of the exchange-repulsion parameters to 30 far-IR and microwave transitions. This IPS constitutes a substantial improvements over the original VRT(ASP-W) potential [11], although van der Avoird and co-workers have obtained one of comparable quality for the $\left(\mathrm{H}_{2} \mathrm{O}\right)_{2}$ isotopomer by "tuning" an ab initio potential derived from symmetry adapted perturbation theory (SAPT) against VRT spectra [18].

The VRT(ASP-W)III yields excellent values for the dimer binding energy and structure (Table 2), as well as the zero-pressure isothermal Joule-Thomson coefficient, $\phi_{0}$ (Figure 1). The zero-pressure Joule-Thomson coefficient is related to the SVC via the equation, $\phi_{0}=B-T * \frac{d B}{d T}$, where $B$ is the

temperature dependent SVC, $T$ is the temperature, and $\frac{d B}{d T}$ is the derivative of $B$ with respect to $T$ [19]. Due to the derivative information inherent within the coefficient, it provides a more stringent test of the IPS than do the second virial coefficients (SVC) themselves. We have recently shown that the quantum corrected SVC's computed from VRT(ASP-W)III also agree nearly perfectly with experimental results over a wide range of temperatures [11]. Combining the data from McGlashan et al. [19] with the values of $\phi_{0}$ calculated from the $B(T)$ results from Kell et al. [20] allows us to test the validity of two-body interaction terms from VRT(ASP-W)III over an even wider range of temperatures. The computed values of $\phi_{0}$ agree very closely with experiment.

\section{Diffusion Quantum Monte Carlo Results for $\left(\mathrm{H}_{2} \mathrm{O}\right)_{2}-\left(\mathrm{H}_{2} \mathrm{O}\right)_{6}$}

The excellent reproduction of dimer properties over such a wide temperature range establishes that VRT(ASP-W)III is an excellent model of 2-body properties of water. Moreover, since it also rigorously represents the leading many-body terms (due to "polarization", or "induction") in terms of a tensorial polarizability and multipole moments, VRT(ASP-W)III can also correctly describe the trimer, tetramer, and larger water clusters.

Much VRT spectroscopic data exist for larger clusters, particularly ground-state properties of up to hexamer [21-26]. We can thus simulate larger clusters with these potentials and compare results to the VRT data in order to further test the validity of our IPS models. DMC is a useful simulation technique for such purposes [27-33]. It is a fully quantum mechanical technique with a computational 
cost that scales favorably with cluster size and potential complexity. Furthermore, it is an excellent complement to $a b$ initio calculations because directly observable vibrationally averaged properties are calculated, rather than just the (unobservable) equilibrium properties. Quack and Suhm have utilized DMC extensively in similar studies of $(\mathrm{HF})_{\mathrm{n}}$ clusters [34-38] and have determined highly accurate potentials for HF aggregates.

The details of our calculations and the corresponding results have been summarized elsewhere [33]. Thus, we only provide a summary of the technique and results below. The starting point of a DMC simulation is the time-dependent Schrödinger equation, which is rewritten in imaginary time and thus becomes isomorphic with the diffusion equation. Consequently, the eigenstate of interest can be simulated via random diffusion. All of the IPS used in the cluster calculations herein use a "frozen monomer" approximation, in which intra-molecular degrees of freedom are not explicitly included in the calculation. Such IPS will be henceforth referred to as rigid potentials. Since intermolecular degrees of freedom are treated separately from intra-molecular vibrations, and are of a lower frequency, a larger time step can be used in the rigid body simulations. A simple method for treating monomers as rigid-bodies has been developed by Buch and others $[39,40]$, called RBDMC, and again the reader is referred to the those references for further information.

Computed equilibrium properties of the trimer through hexamer agree very well with the best available ab initio results (Table 1), and vibrationally averaged ground state structures computed with DMC simulations agree well with experimental data for these clusters (Tables 2 and 3). As shown,VRT(ASP-W)III performs very well as a model for higher order clusters. Upon examination of results for the pentamer and hexamer, it is clear that it represents a substantial improvement over previous fittings [33]. Notably, the DMC calculation on VRT(ASP-W)III correctly predicts that the cage form of the hexamer is the most stable of several low energy structural isomers, as established by Terahertz experiments [41]. As we have noted, this is surprising, considering that VRT(ASP-W)III represents a relatively minor refinement of the previous version in terms of dimer properties. However, this subtle refinement has major global consequences, since VRT(ASP-W)III is a much better model for the larger clusters. Hence, continued fitting of ASP-W or other IPS to larger spectroscopic data sets seems to be a worthwhile pursuit.

The fact that the only many-body force present in VRT(ASP-W)III is induction supports the 
assertion that for gas-phase cluster calculations it seems reasonable to neglect all other many-body contributions (i. e, dispersion, exchange); hence, the computational cost of including 3-body exchange in VRT(ASP-W)III does not seem worthwhile. Given the above encouraging results, we performed classical MC simulations of ambient liquid water to determine how well the dimer-based IPS would perform, recognizing that while the dominant many-body interaction (induction) was well-represented by the ASP-W versions, the much smaller three-body exchange terms were omitted.

\section{Liquid Water Simulations}

Here we employ our VRT(ASP-W)III water dimer potential in Monte Carlo (MC) simulations of bulk liquid water, in order to explore and characterize the nature of the subtle many-body effects that must be added to this polarizable dimer potential in order for it to properly describe bulk properties. We note that because VRT(ASP-W)III contains a tensorial polarizability, it will accurately represent the leading many-body interaction, viz. induction, since the remaining important many-body terms (viz. 3-body exchange, 3-body dispersion) are much smaller.

In the sections below, the results for the radial distribution functions from VRT(ASP-W)III are discussed, and comparison is made with experiments $[42,43]$. The MC simulation technique will not be reviewed, and the reader is directed to [44] and [45] for further information. Comparison is also made to results from Car-Parrinello Molecular Dynamics (CPMD), since these represent a different yet equally valid approach to developing a "first principles" model for liquid water. Also noteworthy is the TTM2-R potential, developed by Burnham and Xantheas [46-49], which accurately models equilibrium properties (i. e., without quantum zero-point effects taken into account) of water clusters as well as structural properties of liquid water and ice. However, TTM2-R reportedly does

not accurately reproduce the water dimer VRT spectrum [50]. Hence, given our ultimate goal of developing a "perfect dimer potential" in order to ascertain the nature of the many-body forces acting within the liquid, results from TTM2-R are not included in the present study. 


\subsection{VRT(ASP-W)III}

\subsubsection{Monte Carlo simulations}

In order to simulate liquid water properties with VRT(ASP-W)III, we used Monte Carlo (MC) rather than Molecular Dynamics (MD) because MC has the advantage of transcending calculations of intermolecular forces, as well as the concomitant disadvantage of not yielding dynamical information. Given the complexity of the IPS, viz. 72 parameters and iterated induction, calculation of the intermolecular forces would be prohibitively expensive. Unless indicated otherwise, our simulations were conducted in the canonical (NVT) ensemble with 64 monomers in a cubic box at ambient density of $1.0 \mathrm{~g} / \mathrm{cm}^{3}$ and a temperature of $25^{\circ} \mathrm{C}$. The simulation was started from an equilibrated MD simulation with the MCY potential. The minimum image convention was applied when calculating distances between center of masses of molecules, and when long range interactions were neglected, interactions were truncated within a cutoff radius equal to half the box length. In order to test the suitability of this cutoff radius, simulations were run at the above density and temperature for systems comprising from 32 to 108 monomers, and the $g\left(R_{O O}\right)$ radial distribution functions (RDF) were already converged at a simulation size of 64 monomers. To insure accurate structural determination from the IPS and adequate simulation times, the results from VRT(ASP-W)III presented below are from simulations of 64 monomers, performed with truncation of the long range forces.

These were run for ca. 5,000 cycles, where one cycle is equal to $N$ Monte Carlo moves, where $N$ is equal to the number of molecules in the simulation, using a well equilibrated liquid structure as the starting point. The minimum image convention was applied when calculating distances between center of masses of molecules. In general, long-range electrostatic interactions in liquid simulations can be handled via Ewald sums, the details of which are found in $[44,45]$, but because ASP-W contains up to a quadrupole on the oxygen and dipoles on the hydrogens, full Ewald sums were too costly to use in these simulations. Simulations of 32 monomers were run with the chargecharge Ewald sums, only, in order to test their importance, and preliminary results indicated that the Ewald sums had little effect on the RDF's computed form VRT(ASP-W)III. Thus, the residual electrostatics were truncated at a cutoff of half the box length. Maximum atomic translational moves of $0.4 \AA$ yielded acceptance ratios of roughly $60 \%$, and molecules were kept rigid via the SHAKE algorithm [51]. Simulations performed with VRT(ASP-W)III had induction and dispersion damped 
with the same hardness factors as for our gas phase calculations [11], and induction was iterated to full convergence.

Comparisons of the calculated RDFs are made to experimental results $[42,43]$ and recently published results from SAPT5s+NB [50,52]. Results for the internal energy of the simulations are shown as well.

\subsubsection{Results}

The oxygen radial distribution function (RDF) computed from VRT(ASP-W)III, shown in Figure 3 , agrees remarkably well with experiment $[42,43]$. The position of the first peak coincides almost exactly with the experimental result, particularly in comparison to neutron diffraction results [43]. More importantly, the $g\left(R_{O O}\right)$ predicts the signature tetrahedral structure of ambient liquid water. The first minimum of the RDF matches almost exactly, and the second maximum occurs at only ca. $0.15 \AA$ too short of a distance. VRT(ASP-W)III does yield a third solvation shell that appears to be contracted relative to experiment. However, the integral of $g\left(R_{O O}\right)$ from the IPS matches those from the experiments very closely, indicating that the number of nearest neighbors in the liquid is correctly described. The $g\left(R_{O H}\right)$ and $g\left(R_{H H}\right)$ from VRT(ASP-W)III agree reasonably well with the experimental results [43]. The IPS produces a liquid phase hydrogen bond length that is ca. $1.9 \AA$, very close to the experimental value.

Comparison of results from VRT(ASP-W)III with those from CPMD are shown in Figure 4. The comparisons are a bit more difficult to make, due to the high degree of variability of the CPMD results. All of the CPMD results were obtained using the BLYP functional, but with varying values for the fictitious electron mass and plane-wave Rydberg cutoff. The results from VRT(ASPW)III for $g\left(R_{O O}\right)$ agree best with the results from [53]. However, as described by Grossman et al. in [8], the use of a high fictitious electron mass in those simulations makes their results somewhat questionable. Conversely, Grossman et al. use a smaller fictitious electron mass (340 vs. $900 \mathrm{au}$ ) and smaller system size ( 32 vs. $\left.64 \mathrm{H}_{2} \mathrm{O}\right)[8]$ and obtain a $g\left(R_{O O}\right)$ that is much more structured than that from either [53] or VRT(ASP-W)III. In order to clarify this issue, we have performed CPMD simulations of $54 \mathrm{H}_{2} \mathrm{O}$ with a small fictitious electron mass of $400 \mathrm{au}$. The RDF's of these new CPMD simulations lie somewhere between the results of [53] and [8], when comparing peak heights 
and minima. This shows that system size effects can be nearly as large as those from choice of the fictitious electron mass. Regardless, all three RDFs from VRT(ASP-W)III compare exceedingly well to these newer CPMD results, although the comparison is not as close as to the results from [53]. However, it is quite possible that these differences would be mitigated through experimentation with different exchange-correlation functionals and pseudo-potentials.

The average potential energy of the ensemble was calculated with VRT(ASP-W)III during the course of our simulations, and the results for $298 \mathrm{~K}$ are shown in Table 4 . The experimental quantity was determined in the standard fashion, by assuming that the water vapor behaves as an ideal gas, subtracting $R T$ from the measured vaporization enthalpy at 298K [54], and multiplying the result by -1. This is equal to the value for the change in internal energy of vaporization $\left(\Delta E_{\text {int }}\right)$, equal to the kinetic plus potential energy of the system. However, because we have assumed the vapor to be an ideal gas (hence, the particles do not interact) and that the temperature of the liquid and vapor are the same (i. e., no change in kinetic energy), $\Delta E_{i n t}=\left\langle E_{p o t}\right\rangle$ of the liquid. As is shown in the table, the value of $\Delta E_{\text {int }}$ from VRT(ASP-W)III is within $20 \%$ of the experimental value, whereas that for SAPT5s+NB deviates from experiment by less than $9 \%$, in the opposite direction. The results from SAPT5s+NB is closer to the experimental value, but the IPS is unable to predict a tetrahedral structure in simulations of ambient liquid water [50]. TTM2-R is able to predict a reasonable liquid water tetrahedral structure, but predicts a value of $\left\langle E_{p o t}\right\rangle$ that is even further from the experimental value as SAPT5s+NB [48]. In addition, it is important to note again that TTM2-R does not have the spectroscopic accuracy of VRT(ASP-W)III.

\subsection{The VRT(MCY) Potentials}

Recently, Leforestier et al. [55] modified the original Clementi MCY potential by adding a fifth site - a floating uncharged virtual site along the $C_{2 v}$ axis, which is then used in place of the oxygen atom in the dispersion terms of the potential. This rigid monomer modified MCY potential was then fit to $\left(\mathrm{H}_{2} \mathrm{O}\right)_{2}$ data, and shown to reproduce experimentally determined ground-state properties and excited intermolecular vibrational state band origins very well. The resulting IPS was named VRT(MCY-5r), where the "VRT" corresponds to the vibration-rotational-tunneling data to which it was fit, and " $5 \mathrm{r}$ " corresponds to the 5 sites of the rigid monomer potential. 
In an effort to include many-body interactions in the above potential, a functional form developed by Bernardo et al. [56], containing ab initio atomic polarizabilities, was added to model the manybody induction energy [57]. This new potential was named VRT(MCY-5r/pol), where "pol" refers to the fact that the water monomers have polarizable sites. While this IPS is also able to reproduce experimental observables of the water dimer to a high degree of accuracy [57], DMC calculations performed by us show that both of the above potentials yield inaccurate ground-state structural properties for $\left(\mathrm{H}_{2} \mathrm{O}\right)_{2}$. For example, both of the above IPS yield vibrational ground-state average O-O distances between the monomers, $\left\langle R_{O O}\right\rangle$, that are between 3.10 and $3.20 \AA$, compared to the experimental value of $2.99 \AA$ [58]. Hence, in terms of modeling clusters larger than the dimer or of modeling the liquid, this IPS does not have a sufficient level of accuracy. However, VRT(MCY$5 \mathrm{r} / \mathrm{pol}$ ) is included in the present discussion because of its relatively simple functional form, and since it provides helpful comparison to results from the SAPT5s+NB IPS.

\subsubsection{Molecular Dynamics simulations}

Due to its simplicity, VRT(MCY-5r/pol) could be simulated via MD. This had the advantage of allowing us to determine dynamical as well as structural information about the potential. Due to the fact that the IPS has electrostatic terms no larger than the dipole, it was possible to use full Ewald sums in the simulations. The minimum image convention was applied when calculating distances between center of masses of molecules, and all short range interactions were truncated within a cutoff radius equal to half the box length. Our simulations were conducted in the canonical (NVT) ensemble with $32-256$ monomers in a cubic box at ambient density of $1.0 \mathrm{~g} / \mathrm{cm}^{3}$ and a temperature of $25^{\circ} \mathrm{C}$.

This section describes our Molecular Dynamics simulation results from VRT(MCY-5r/pol). The liquid structure was found to saturate at simulation sizes of 108 monomers, and results from 256 monomer simulations are presented herein. A time step of $0.5 \mathrm{fs}$ was used for the intermolecular force field for both IPS, and all simulations were run for a total of 10 ps. 


\subsubsection{Results}

The RDFs for VRT(MCY-5r/pol) are shown in Figure 5, with comparison made to results from SAPT5s+NB [50] and experiment [42,43]. For VRT(MCY-5r/pol), the first peak of the $g\left(R_{O O}\right)$ occurs at too large distance (ca. 10\%), which is to be expected considering the overestimation of the dimer vibrational ground-state $\left\langle R_{O O}\right\rangle$ by the IPS. Nonetheless, for $g\left(R_{O O}\right)$, the result does not exhibit the requisite second (tetrahedral) peak at ca. $4.5 \AA$, and interestingly resembles the RDF of a Lennard-Jones fluid. In addition, there exists a clearly visible but small third solvation shell at ca. $9 \AA$ (not shown for the sake of clarity). The $g\left(R_{O H}\right)$ has too small of a first peak (ca. $20 \%$ too low) and predicts a hydrogen bond length of $2 \AA$. However, the second peak that matches reasonably well with experiment. Finally, the shape of the $g\left(R_{H H}\right)$ matches the experimental results well, although the two peaks are slightly too close together, as was the case for VRT(ASP-W)III. Also, both $g\left(R_{O H}\right)$ and $g\left(R_{H H}\right)$ of VRT(MCY-5r/pol) exhibit the third solvation shell at ca. $6 \AA$.

Also interesting is the fact that these results are very similar to those from SAPT5s+NB. The maximum of the first peak from SAPT5s +NB is closer to the experimental value, but it is similarly too wide and the $g\left(R_{O O}\right)$ also lacks the second maximum at $4.5 \AA$. Thus, this behavior appears to be a general characteristic of "spectroscopic" potentials with functional forms simpler than that of ASP-W, used to model the liquid. Interestingly, integration of the plots of $g\left(R_{O O}\right)$ in Figure 5 shows that the RDFs from both VRT(MCY-5r/pol) and SAPT5s+NB yield cumulative sums that are strikingly similar to that of the experimental curve. This implies that all three results yield roughly the same number of nearest neighbors, when comparing the values of the integral up to the first minimum, despite the fact that VRT(MCY-5r/pol) and SAPT5s + NB do not yield tetrahedral liquid structure.

The IPS was further assessed through calculation of the mean squared displacement of the center of mass of each monomer, $\left\langle R_{C M}^{2}(t)\right\rangle$. From this, the diffusion constant $(D)$ can be determined by calculating the slope of the curve in Figure 6. The plot shows yet another remarkable feature, in that the potential yields a diffusion constant of zero (for the time scale of our simulation). In order to get the monomers simulated on the VRT(MCY-5r/pol) surface to diffuse on the time scales of our calculations, these simulations were run at temperature as high as $598 \mathrm{~K}$, yet no significant change was found in the $\left\langle R_{C M}^{2}(t)\right\rangle$ curve or the RDFs. 


\section{Discussion}

The recently developed polarizable, 6D water dimer potential, VRT(ASP-W)III, has been tested in simulations of liquid water at ambient conditions. We have shown that this spectroscopic "first principles" polarizable dimer potential reproduces the vibrational ground-state structures of the trimer through hexamer as well as the detailed vibration-rotational tunneling states of the dimer, and thus is a good start toward developing a "universal potential" for water. VRT(ASP-W)III is able to produce a $g\left(R_{O O}\right)$ reflecting the tetrahedral structure characteristic of liquid water. The $g\left(R_{O H}\right)$ and $g\left(R_{H H}\right)$ calculated from the IPS also compare favorably to experiments [43] in terms of peak positions, but for $g\left(R_{O H}\right)$, the first peak is still too low and the second peak too high; for $g\left(R_{H H}\right)$, the peaks are too close together. Interestingly, the results from two other spectroscopic surfaces, VRT(MCY-5r/pol) and SAPT5s+NB, do not predict tetrahedral liquid water structure, and yet both IPS predict $g\left(R_{O H}\right)$ and $g\left(R_{H H}\right)$ functions that compare well with experiments. It should be noted again that simulations with all three rigid, "spectroscopic" potentials exhibit a number of nearest neighbors within the first solvation shell that compares very well with experiment (within ca. $5 \%$ ), despite the fact that the much simpler SAPT5s+NB and VRT(MCY-5r) IPS yield inaccurate liquid structures. The most striking differences between VRT(ASP-W)III and the previous two IPS is that it was fit to a much larger set of experimental data. This gives clear testament to the fact that exact determination of the water dimer IPS is a very subtle process, wherein small changes in the potential can have large ramifications for the predicted properties of condensed phases.

It is interesting to note once again that Burnham and Xantheas were able to achieve some level of 'universality' in their TTM2-R potential, by fitting a 6-10-12 Lennard-Jones functional form to only 25 very high level $a b$ initio points calculated along the $C_{s}$ symmetry. While the potential is highly accurate for geometries within that symmetry, it does not have the same level of accuracy for the energetics of others (viz. $C_{2}, C_{i}$, and $C_{2 v}$ ) [48], and as we note above, is not an accurate model for the dimer VRT spectrum. However, it does yield good results for those bulk properties tested by Burnham and Xantheas [48]. Hence, it would be very interesting to refine their potential via comparison to experimental data, to see what effect (positive or negative) this would have on the accuracy of its structural predictions for liquid water and ice. In addition, Burnham and Xantheas have developed the TTM2-F potential, which includes both intramolecular flexibility and a monomer 
geometry dependent polarization [49]. TTM2-F yields highly accurate water cluster equilibrium, non-quantum corrected energetics and accurately describes monomer geometry deformation with increasing cluster size, and thus appears to achieve a higher level of 'universality' than its predecessor, TTM2-R. Consequently, it would be interesting to investigate its predictive powers in terms of the dimer VRT spectrum and larger cluster vibrational ground-states.

The value $\Delta E_{\text {int }}$ yielded from VRT(ASP-W)III is too low, and could easily be due to the exclusion of nuclear quantum effects in our simulations. Treatment of quantum effects in liquid simulations is beyond the scope of this paper, and the reader is referred to [59-61] for discussions. Nonetheless, quantum effects in liquid water are generally regarded as being fairly small, and a rule of thumb is that they are equivalent to raising the temperature of a simulation by $50^{\circ} \mathrm{C}[4,5]$. However, it is quite possible that the quantum effects in the liquid will be far more dramatic when using a spectroscopic potential, such as VRT(ASP-W)III, that is derived from an explicitly quantum dynamical model. In an empirical liquid water potential, a classical simulation is used to fit the potential to experimental results. Consequently, any potential energy barrier between configurations will be artificially flattened in order to effectively allow classical mechanics to represent tunneling effects and zero-point effects. As a result, it is not surprising that such potentials exhibit small apparent quantum effects. However, a "spectroscopic" potential, formulated from first-principles, will not exhibit the same artificial lowering of tunneling barriers. Hence, tunneling effects in the liquid could be significant, as could familiar zero-point effects. This should be explored in future work.

\section{Acknowledgments}

This work was supported by the Experimental Physical Chemistry Program of the National Science Foundation. The authors thank Larry Fried of LLNL for many helpful discussions. R.J.S. and C.F. gratefully acknowledge a grant from the Integrated Programs Division of the NSF.

This work was performed under the auspices of the U.S. Department of Energy by the University of California, Lawrence Livermore National Laboratory under Contract No. W-7405-Eng-48. 


\section{References}

[1] B. Guillot, J. Mol. Liq. 101, 219 (2003).

[2] W. L. Jorgensen, J. Chandrasekhar, J. D. Madura, R. W. Impey, and M. L. Klein, J. Chem. Phys. 79, 926 (1983).

[3] H. J. C. Berendsen, J. R. Grigera, and T. P. Straatsma, J. Phys. Chem. 91, 6269 (1987).

[4] R. A. Kuharsky and P. J. Rossky, J. Chem. Phys. 82, 5164 (1985).

[5] G. S. Del Buono, P. J. Rossky, and J. Schnitker, J. Chem. Phys. 95, 3728 (1991).

[6] A. J. Stone, The Theory of Intermolecular Forces, The International Series of Monographs on Chemistry (Clarendon Press, Oxford, 1996).

[7] S. Rybak, B. Jeziorski, and K. Szalewicz, J. Chem. Phys. 95, 6576 (1991).

[8] J. Grossman, E. Schwegler, E. W. Draeger, F. Gygi, and G. Galli, J. Chem. Phys. 120, 300 (2004).

[9] M. P. Hodges, A. J. Stone, and S. S. Xantheas, J. Phys. Chem. A 101, 9163 (1997).

[10] L. Ojamäe and K. Hermansson, J. Phys. Chem. 98, 4271 (1994).

[11] N. Goldman, R. S. Fellers, M. G. Brown, L. B. Braly, C. J. Keoshian, C. Leforestier, and R. J. Saykally, J. Chem. Phys. 116, 10148 (2002), and references therein.

[12] C. Millot and A. J. Stone, Mol. Phys. 77, 439 (1992).

[13] R. J. Saykally and G. A. Blake, Science 259, 1570 (1993).

[14] L. B. Braly, J. D. Cruzan, K. Liu, R. S. Fellers, and R. J. Saykally, J. Chem. Phys. 112, 10293 (2000).

[15] L. B. Braly, K. Liu, M. G. Brown, F. N. Keutsch, R. S. Fellers, and R. J. Saykally, J. Chem. Phys. 112, 10314 (2000). 
[16] K. L. Busarow, R. C. Cohen, G. A. Blake, K. B. Laughlin, Y. T. Lee, and R. J. Saykally, J. Chem. Phys. 90, 3937 (1989).

[17] R. S. Fellers, C. Leforestier, L. Braly, M. G. Brown, and R. J. Saykally, Science 284, 945 (1999).

[18] G. C. Groenenboom, E. Mas, R. Bukowski, K. Szalewicz, P. E. S. Wormer, and A. van der Avoird, Phys. Rev. Lett. 84, 4072 (2000).

[19] M. L. McGlashan and C. J. Wormald, J. Chem. Therm. 32, 1489 (2000).

[20] G. S. Kell, G. E. McLaurin, and E. Whalley, Proc. R. Soc. Lond. A 425, 49 (1989).

[21] K. Liu, J. G. Loeser, M. J. Elrod, B. C. Host, J. A. Rzepiela, and R. J. Saykally, J. Am. Chem. Soc. 116, 3507 (1994).

[22] K. Liu, J. D. Cruzan, and R. J. Saykally, Science 271, 929 (1996), and references therein.

[23] K. Liu, M. G. Brown, C. Carter, R. J. Saykally, J. K. Gregory, and D. C. Clary, Nature 381, 501 (1996).

[24] K. Liu, M. G. Brown, J. D. Cruzan, and R. J. Saykally, Science 271, 62 (1996).

[25] K. Liu, M. G. Brown, and R. J. Saykally, J. Phys. Chem. A 101, 8995 (1997).

[26] N. Pugliano and R. J. Saykally, Science 257, 1937 (1992).

[27] J. Gregory and D. C. Clary, Chem. Phys. Lett. 228, 547 (1994).

[28] J. K. Gregory and D. C. Clary, J. Chem. Phys. 102, 7817 (1995).

[29] J. Gregory and D. C. Clary, J. Chem. Phys. 103, 8924 (1995).

[30] J. K. Gregory and D. C. Clary, J. Phys. Chem 100, 18014 (1996).

[31] J. K. Gregory and D. C. Clary, J. Chem. Phys. 105, 6626 (1996).

[32] J. K. Gregory, D. C. Clary, K. Liu, M. G. Brown, and R. J. Saykally, Science 275, 814 (1997).

[33] N. Goldman and R. J. Saykally, J. Chem. Phys. 120, 4777 (2004). 
[34] M. Quack and M. A. Suhm, Mol. Phys. 69, 79‘ (1990).

[35] M. Quack and M. A. Suhm, Chem. Phys. Lett. 183, 187 (1991).

[36] M. Quack and M. A. Suhm, J. Chem. Phys. 95, 28 (1991).

[37] W. Kloppera, M. Quack, and M. A. Suhm, J. Chem. Phys. 108, 10096 (1998).

[38] M. Quack, R. Stohner, and M. A. Suhm, J. Mol. Struct. 599, 381 (2001).

[39] V. Buch, J. Chem. Phys. 97, 726 (1992).

[40] P. Sandler, J. O. Jung, M. M. Szczesniak, and V. Buch, J. Chem. Phys. 101, 1378 (1994).

[41] F. N. Keutsch and R. J. Saykally, Proc. Natl. Acad. Sci. U.S.A. 98, 10533 (2001).

[42] G. Hura, J. M. Sorenson, R. M. Glaser, and T. Head-Gordon, J. Chem. Phys. 113, 9140 (2000).

[43] A. K. Soper, Chem. Phys. 258, 121 (2000).

[44] M. P. Allen and D. J. Tildesley, Computer Simulations of Liquids (Clarendon Press, Oxford, 1989).

[45] D. Frenkel and B. Smit, Understanding Molecular Simulation (Academic Press, San Diego, 1996).

[46] C. J. Burnham and S. Xantheas, J. Chem. Phys. 116, 1479 (2002).

[47] S. Xantheas, C. J. Burnham, and R. J. Harrison, J. Chem. Phys. 116, 1493 (2002).

[48] C. J. Burnham and S. Xantheas, J. Chem. Phys. 116, 1500 (2002).

[49] C. J. Burnham and S. Xantheas, J. Chem. Phys. 116, 5115 (2002).

[50] E. M. Mas, R. Bukowski, and K. Szalewicz, J. Chem. Phys. 118, 4404 (2003), and references therein.

[51] J.-P. Ryckaert, G. Ciccotti, and H. J. C. Berendsen, J. Comp. Phys. 23, 327 (1977).

[52] E. M. Mas, R. Bukowski, and K. Szalewicz, J. Chem. Phys. 118, 4386 (2003). 
[53] P. L. Silvestrelli and M. Parrinello, Phys. Rev. Lett. 82, 3308 (1999).

[54] N. E. Dorsey, Properties of Ordinary Water-Substance (Hafner, New York, 1968).

[55] C. Leforestier, F. Gatti, R. S. Fellers, and R. J. Saykally, J. Chem. Phys. 117, 8710 (2002).

[56] D. N. Bernardo, Y. Ding, K. Krogh-Jespersen, and R. M. Levy, J. Phys. Chem. 98, 4180 (1994).

[57] C. Leforestier, N. Goldman, and R. J. Saykally, (in preparation).

[58] T. R. Dyke, K. M. Mack, and J. S. Muenter, J. Chem. Phys. 66, 498 (1977).

[59] R. P. Feynman and A. R. Hibbs, Quantum Mechanics and Path Integrals (McGraw-Hill, New York, 1965).

[60] L. S. Schulman, Techniques and Applications of Path Integration (Wiley, New York, 1981).

[61] R. P. Feynman, Staistical Mechanics (Addison-Wesley, Reading, Massachusetts, 1998).

[62] A. J. Stone, A. Dullweber, O. Engkvist, E. Fraschini, M. P. Hodges, A. W. Meredith, P. L. A. Popelier, and D. J. Wales, Orient: A Program for Studying Interactions Between Molecules, Version 4.4, University of Cambridge, 2000, http://fandango.ch.cam.ac.uk/.

[63] L. B. Braly, Ph.D. thesis, University of California, Berkeley, 1999. 


\section{Tables}

\begin{tabular}{ccc}
\hline \hline & VRT(ASP-W)III & ab initio \\
\hline$\left(\mathrm{H}_{2} \mathrm{O}\right)_{2}$ & -4.96 & -4.98 \\
$\left(\mathrm{H}_{2} \mathrm{O}\right)_{3}$ & -15.53 & -15.8 \\
$\left(\mathrm{H}_{2} \mathrm{O}\right)_{4}$ & -28.74 & -27.6 \\
$\left(\mathrm{H}_{2} \mathrm{O}\right)_{5}$ & -38.31 & -36.3 \\
$\left(\mathrm{H}_{2} \mathrm{O}\right)_{6}$ cage & -48.80 & -45.8 \\
$\left(\mathrm{H}_{2} \mathrm{O}\right)_{6}$ prism & -49.97 & -45.9 \\
\hline \hline
\end{tabular}

Table 1: Comparison of minimum energy structures to best available ab initio results [48]. Equilibrium structures from VRT(ASP-W)III were calculated by the Orient program [62]. Results from VRT(ASP-W)III are very close to the ab initio energetic ordering of results [33]. More importantly, the IPS predicts the correct energetic ordering of the hexamer prism and cage structures. This ordering is reversed when quantum zero-point energies are taken into account [33]. 


\begin{tabular}{cccc}
\hline \hline \multicolumn{4}{c}{ Equilibrium AND Ground-STATE WaTER Dimer PROPERTIES } \\
& Expt. & VRT(ASP-W)III & VRT(ASP-W)III $_{e q}$ \\
\hline$D_{e}[\mathrm{kcal} / \mathrm{mol}]$ & $5.40(0.5)$ & - & 4.96 \\
$D_{0}[\mathrm{kcal} / \mathrm{mol}]$ & $3.66(0.5)$ & 3.09 & - \\
$R_{O O} \AA$ & $2.976(0.00,-0.03)$ & 3.01 & 2.95 \\
$\mathrm{~A}[\mathrm{GHz}]$ & 227.6 & 230.36 & 220.06 \\
$(\mathrm{~B}+\mathrm{C}) / 2[\mathrm{GHz}]$ & 6.16 & 5.96 & 6.24 \\
$\mathrm{~B}-\mathrm{C}[\mathrm{MHz}]$ & 33.11 & 70 & - \\
\hline \hline
\end{tabular}

Table 2: Water dimer equilibrium and vibrational ground-state properties from VRT(ASP-W)III, with comparison to experiment [41]. Using these data in conjunction with measurements for other dimer isotopes, they derived the equilibrium structure parameter, $R_{O O}$. The experimental observables, $\mathrm{A},(\mathrm{B}+\mathrm{C}) / 2$, and $\mathrm{B}-\mathrm{C}$ were taken from ref. [63], p. 130 and 170. As stated in the text, the VRT(ASP-W)III vibrationally averaged properties were determined by DMC. The rotational constants were calculated using fixed monomer geometries of $R_{O H}=0.9572$ and $\angle \mathrm{HOH}=104.52^{0}$, and masses $\mathrm{H}=1.0070511 \mathrm{au}$ and $\mathrm{O}=15.994915 \mathrm{au}$. The equilibrium properties from VRT(ASPW)III, listed above, were calculated by the Orient molecular interaction program, from Stone and co-workers [62]. 


\begin{tabular}{c|ccc|cc}
\hline \hline$\left(\mathrm{H}_{2} \mathrm{O}\right)_{3}$ & VRT(ASP-W)III & Experiment & $\left(\mathrm{D}_{2} \mathrm{O}\right)_{4}$ & VRT(ASP-W)III & Experiment \\
\hline $\mathrm{A}(\mathrm{GHz})$ & 6.84 & 6.65 & $\mathrm{~A}(\mathrm{GHz})$ & 3.18 & 3.08 \\
$\mathrm{~B}(\mathrm{GHz})$ & 5.68 & 6.65 & $\mathrm{~B}(\mathrm{GHz})$ & 2.77 & 3.08 \\
$\mathrm{C}(\mathrm{GHz})$ & 3.15 & $3.51(\mathrm{AF})$ & $\mathrm{C}(\mathrm{GHz})$ & 1.54 & $(\mathrm{AF})$ \\
\hline \hline
\end{tabular}

\begin{tabular}{c|ccc|cc}
\hline \hline$\left(\mathrm{D}_{2} \mathrm{O}\right)_{5}$ & VRT(ASP-W)III & Experiment & $\left(\mathrm{H}_{2} \mathrm{O}\right)_{6}$ & VRT(ASP-W)III & Experiment \\
\hline $\mathrm{A}(\mathrm{GHz})$ & 1.83 & 1.75 & $\mathrm{~A}(\mathrm{GHz})$ & 2.14 & 2.16 \\
$\mathrm{~B}(\mathrm{GHz})$ & 1.60 & 1.75 & $\mathrm{~B}(\mathrm{GHz})$ & 1.07 & 1.13 \\
$\mathrm{C}(\mathrm{GHz})$ & 0.85 & $(\mathrm{AF})$ & $\mathrm{C}(\mathrm{GHz})$ & 1.04 & 1.07 \\
\hline \hline
\end{tabular}

Table 3: Rotational constants from VRT(ASP-W)III for the water trimer through hexamer, with comparison to experiment. The label of AF indicates that the $\mathrm{C}$ rotational constant could not be measured for a given cluster, and thus its value was "arbitrarily fixed." $\mathrm{H}_{2} \mathrm{O}$ results are not available for the tetramer and pentamer, so $\mathrm{D}_{2} \mathrm{O}$ results are presented instead.

\begin{tabular}{c|c|c|c|c} 
& VRT(ASP-W)III & SAPT5s+NB & TTM2-R & Expt. \\
\hline$\left\langle E_{p o t}\right\rangle[\mathrm{kJ} / \mathrm{mol}]$ & -51.46 & -45.02 & -46.90 & -41.51
\end{tabular}

Table 4: $\Delta E_{\text {int }}$ at $298 \mathrm{~K}$, with comparison to the experimental value from [54]. The results from SAPT5s+NB is closer to the experimental value, but the IPS is unable to predict a tetrahedral structure in simulations of ambient liquid water [50]. TTM2-R is able to predict a reasonable liquid water tetrahedral structure, but predicts a value of $\left\langle E_{\text {pot }}\right\rangle$ that is even further from the experimental value as SAPT5s+NB [48]. In addition, it is important to note that TTM2-R does not have the spectroscopic accuracy of VRT(ASP-W)III. 


\section{Figures}

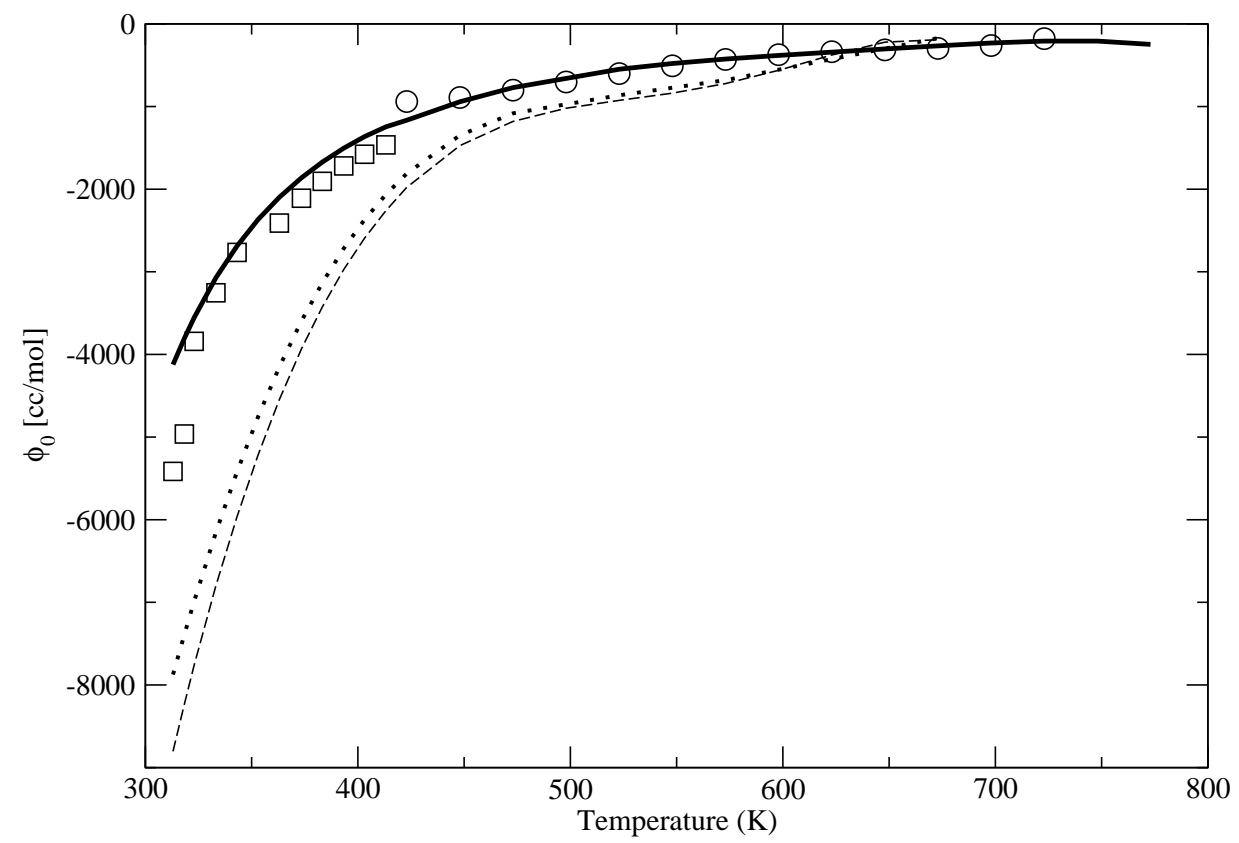

Figure 1: Joule-Thomson coefficient for $\left(\mathrm{H}_{2} \mathrm{O}\right)_{2}$ from VRT(ASP-W)III, with comparison to experiment. The solid black line corresponds to VRT(ASP-W)III, the open squares to experimental results from [19], the open circles to experimental results from [20], the dotted line to ASP-W [12], and the dashed line to TIP4P [2]. The discrepancies evident at lower temperatures are due to the truncation of quantum effects in the calculated SVC to first order. 


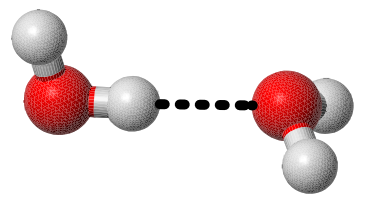

(a) Dimer

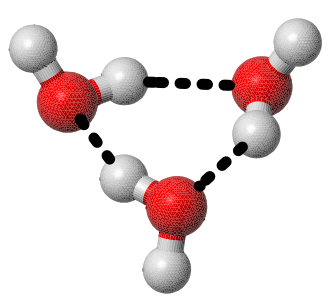

(b) Trimer (uud)

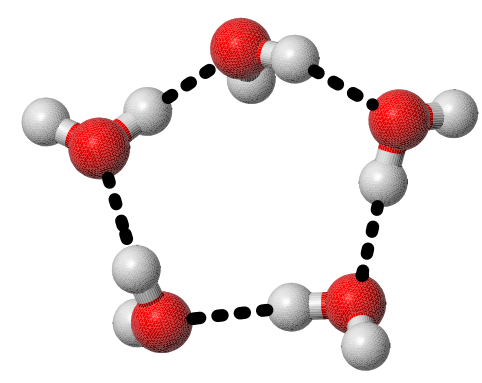

(d) Cyclic

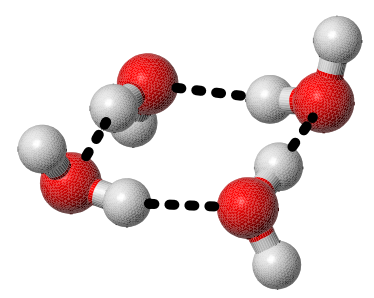

(c) Tetramer (udud)

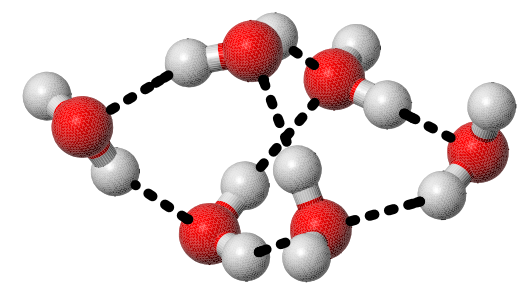

(e) Cage

Figure 2: Vibrational ground-state structures from VRT(ASP-W)III. 


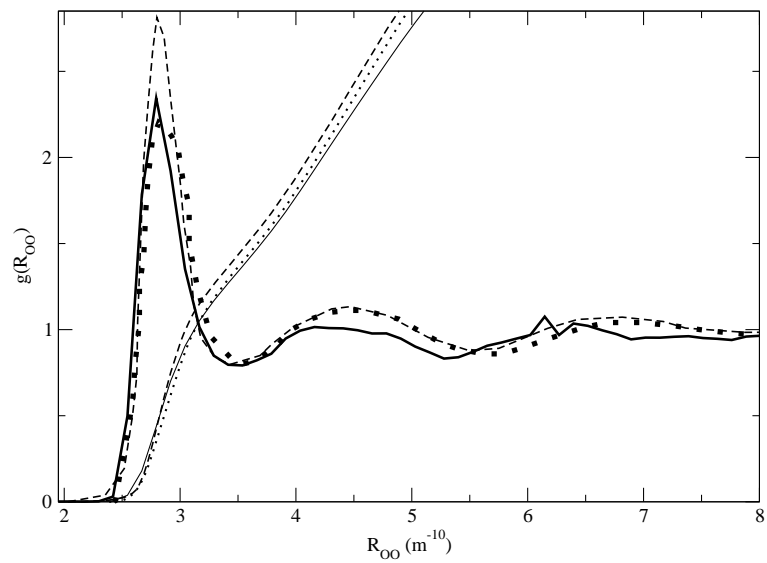

(a)

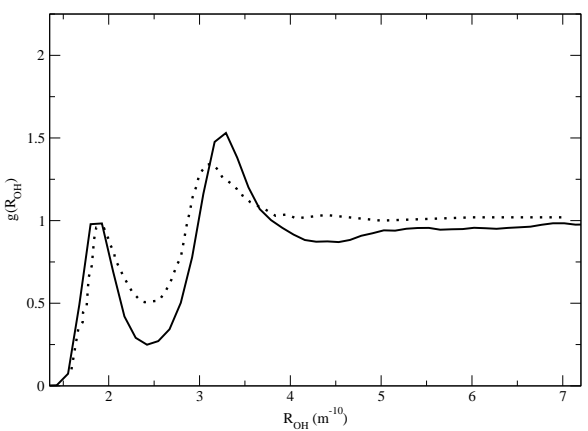

(b)

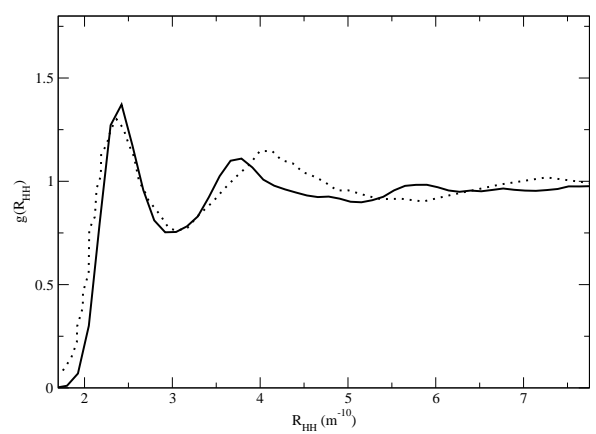

(c)

Figure 3: (a) $g\left(R_{O O}\right)$, (b) $g\left(R_{O H}\right)$, and (c) $g\left(R_{H H}\right)$, for VRT(ASP-W)III, with comparison to experiment [42, 43]. The solid curves corresponds to VRT(ASP-W)III, the dotted curves to [43], and the dashed curve to [42]. Superimposed upon the $g\left(R_{O O}\right)$ plot are the respective cumulative integrals of the RDFs. Simulations were conducted in the canonical (NVT) ensemble with 64 monomers in a cubic box at ambient density of $1.0 \mathrm{~g} / \mathrm{cm}^{3}$ and a temperature of $25^{\circ} \mathrm{C}$. In order to extend the RDF to the end of the simulation box, the molecular configurations were duplicated in all directions, resulting in 512 configurations per time step, when calculating this property. 


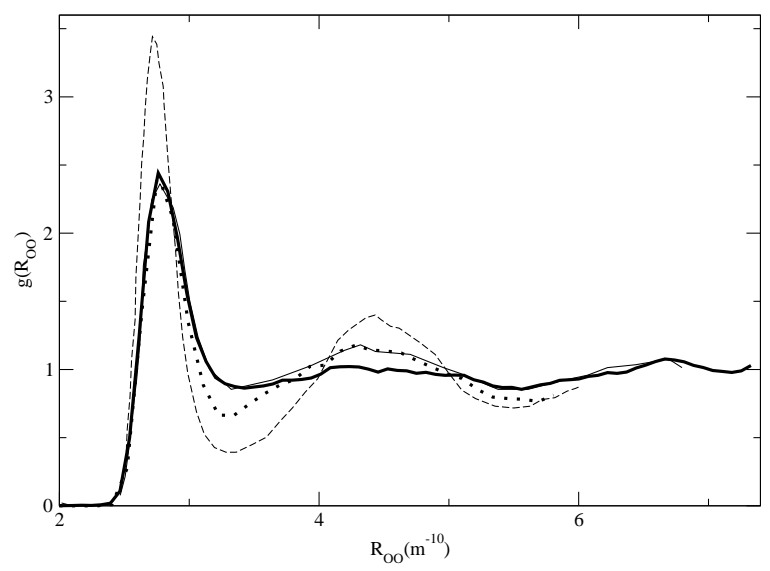

(a)

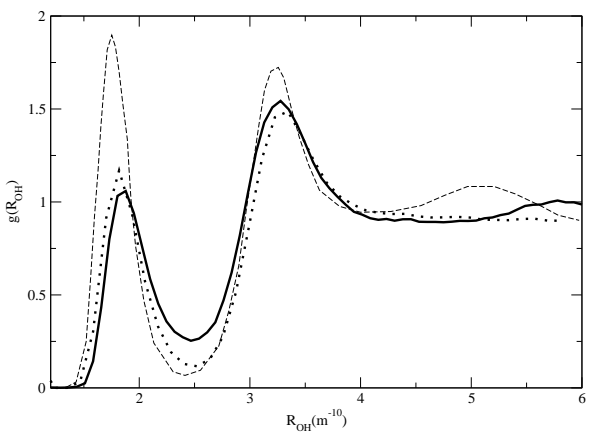

(b)

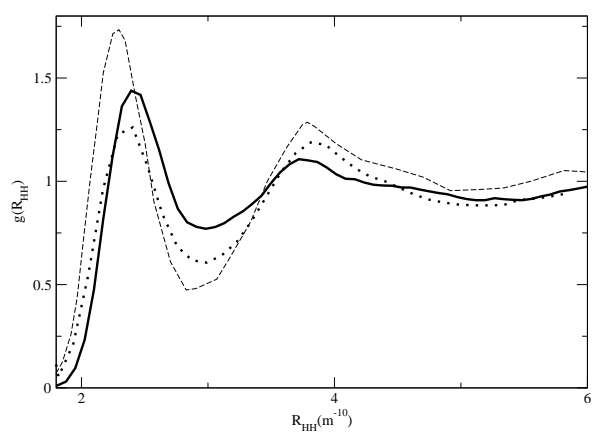

(c)

Figure 4: (a) $g\left(R_{O O}\right)$, (b) $g\left(R_{O H}\right)$, and (c) $g\left(R_{H H}\right)$, for ambient water, from VRT(ASP-W)III and with comparison to results from CPMD $[8,53]$. The thick solid lines correspond to VRT(ASP-W)III, the thin $g\left(R_{o o}\right)$ line to [53], the dashed lines to [8], and the dotted lines to our own CPMD simulations. It is important to note that the results from [53] have a simulation size of $64 \mathrm{H}_{2} \mathrm{O}$, but use an exceedingly high fictitious electron mass of 900 au and a low Rydberg cutoff of 70 . In contrast, the results from [8] use a fictitious mass of only 340 au., but have an exceedingly small system size of $32 \mathrm{H}_{2} \mathrm{O}$, and a moderate energetic cutoff of 85 Rydberg. Our CPMD results have a simulation size of $54 \mathrm{H}_{2} \mathrm{O}$ and use a fictitious electron mass of 400 au and an energetic cutoff of 85 Rydberg. 


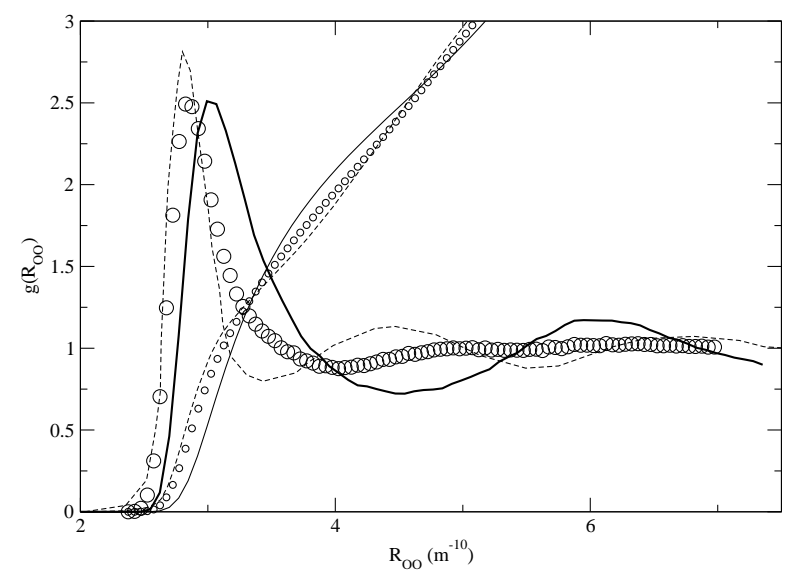

(a)

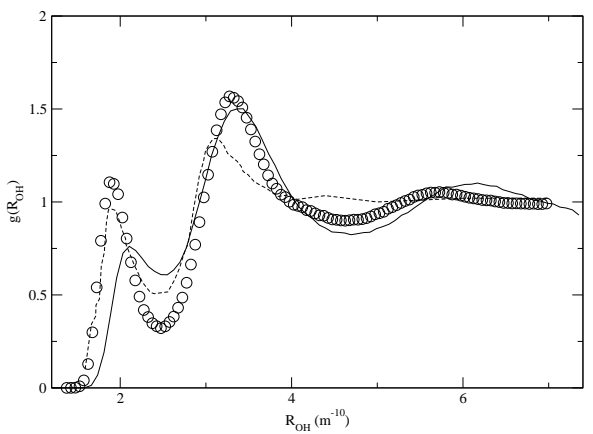

(b)

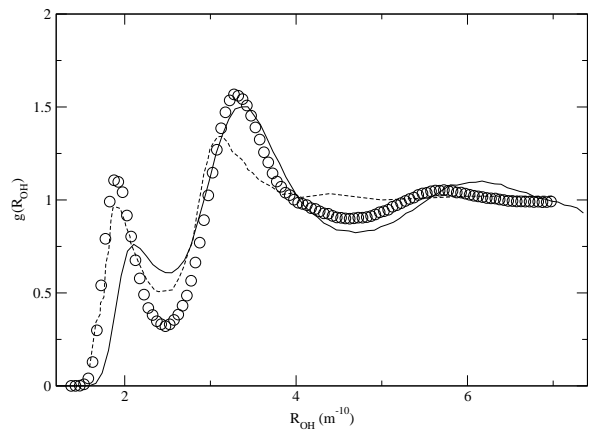

(c)

Figure 5: (a) $g\left(R_{O O}\right)$, (b) $g\left(R_{O H}\right)$, and (c) $g\left(R_{H H}\right)$, for VRT(MCY-5r/pol), with comparison to SAPT5s+NB [50] and experiment [42]. The solid curves corresponds to VRT(MCY-5r/pol), the open circles to [50] and the dashed curve to [42]. Again, superimposed upon the $g\left(R_{O O}\right)$ plot are the respective cumulative integrals of the RDFs. Simulations were conducted in the canonical (NVT) ensemble with 108 monomers in a cubic box at ambient density of $1.0 \mathrm{~g} / \mathrm{cm}^{3}$ and a temperature of $25^{\circ} \mathrm{C}$. 


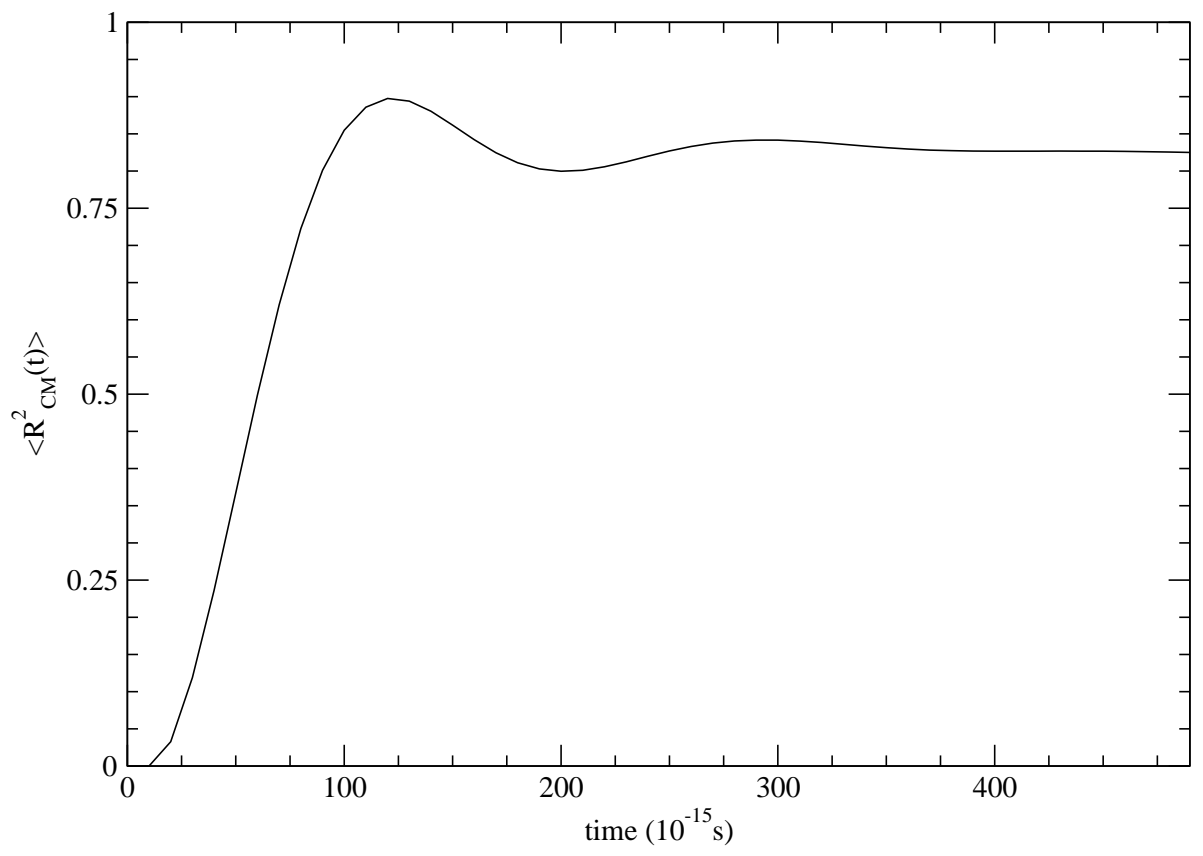

Figure 6: $\left\langle R_{C M}^{2}(t)\right\rangle$ for $\operatorname{VRT}(\mathrm{MCY}-5 \mathrm{r} / \mathrm{pol})$ [solid line] at $298 \mathrm{~K}$. The slope of the curve at $t \gg 0$ yields the value of the diffusion constant, $D$. 